\title{
THE ASTROPHYSICAL JOURNAL
}

VOLUME 139

JANUARY 1, 1964

NUMBER 1

\section{AN ANALYSIS OF THE SPECTRUM OF MARS}

Lewis D. Kaplan, Guido Münch, and Hyron SpinRad

Space Sciences Division, Jet Propulsion Laboratory and Mount Wilson and Palomar Observatories

Carnegie Institution of Washington, California Institute of Technology

Received November 11, 1963

\section{ABSTRACT}

On a high-dispersion spectrogram of Mars taken at Mount Wilson rotational lines of $\mathrm{H}_{2} \mathrm{O}$ near $\lambda 8300$ and $\mathrm{CO}_{2}$ near $\lambda 8700$ have been detected. Recent laboratory measurements of line strengths by D. Rank have been used to determine the amounts of $\mathrm{H}_{2} \mathrm{O}$ and $\mathrm{CO}_{2}$ in the atmosphere of Mars: $14 \pm 7 \mu$ precipitable water and $55 \pm 20 \mathrm{~m}$ atm $\mathrm{CO}_{2}$. From the absence of $\mathrm{O}_{2}$ in the Martian spectra, we set an upper limit of $70 \mathrm{~cm}$ atm for the $\mathrm{O}_{2}$ content.

By suitably combining the $\mathrm{CO}_{2}$ amount with observations by Kuiper and Sinton of the strongly saturated bands in the $2-\mu$ region, a surface pressure of $25 \pm 15 \mathrm{mb}$ has been derived.

The implications of the results on the composition of the Martian atmosphere are discussed.

\section{INTRODUCTION}

The polar caps of Mars have been found by Kuiper (1952) to have an infrared reflection spectrum consistent with a thin layer of $\mathrm{H}_{2} \mathrm{O}$ frost. The polarization of the light reflected by the polar caps as observed and interpreted by Dollfus (1961) has also provided evidence for the existence of $\mathrm{H}_{2} \mathrm{O}$ ice on the surface of Mars. At the vapor pressures estimated to prevail in that planet, phase transitions of $\mathrm{H}_{2} \mathrm{O}$ will be from ice to vapor. Moreover, the seasonal variations observed in the extent of the Martian polar caps imply that there is a net transport of $\mathrm{H}_{2} \mathrm{O}$ between both poles, and that sometime during the cycle the atmosphere must contain in vapor form a significant fraction of the amount that may be present in the form of ice. De Vaucouleurs (1954) has estimated on this basis "an atmospheric water vapor content of the order of 0.1 mm or less." From the sublimation rate of a polar cap and its temperature Sagan (1961) has suggested the value of $10^{-2} \mathrm{~mm}$ for the same quantity. An amount of this order of magnitude suffices, according to Hess (1948), to explain the nocturnal and sunrise limb clouds observed on Mars.

In contrast with these indirect estimates stand a number of unsuccessful attempts to detect spectroscopically water vapor in Mars. From Mount Wilson observations gathered in 1933 and 1937-39 (Adams 1943), Dunham (1952), for example, concluded: "it seems unlikely that there is more than 0.0015 as much water vapor above the surface of Mars as there is above Mt. Wilson on an average clear night in Winter." This would be some $10 \mu$ of precipitable $\mathrm{H}_{2} \mathrm{O}$ on Mars. The arguments raised by Hess (1951) diminish the precision given by Dunham to this upper limit, but they do not explain why the results of the observations were negative. The more recent observation by Kiess, Corliss, Kiess, and Corliss (1957) has been equally negative, although the upper 
limit to the water-vapor content of Mars fixed by these authors through a comparison with absorption-cell spectra is nearly ten times higher than Dunham's. Further efforts to settle the question are obviously worthwhile, especially since the possibility that water may be contained in the Martian atmosphere only in the form of ice crystals has been suggested by Lebedinskii and Salova (1962). But in order to have some hopes of success it appeared to us that a considerable increase in spectral resolving power over previous attempts was required. The proper consideration of the curve of growth for the strongest lines in the $(2,1,1)$ band of $\mathrm{H}_{2} \mathrm{O}$ at $\lambda 8200 \AA$ established that a column of only $10 \mu$ of $\mathrm{H}_{2} \mathrm{O}$, at the pressure generally adopted for Mars's atmosphere, should produce lines a few milliangstroms strong (Spinrad and Richardson 1963). Under observational conditions of low humidity and near the time of the maximum Doppler velocity between Earth and Mars, such lines should be detectable using the highest resolving power available at the 100-inch coudé spectrograph and a high-contrast emulsion. The required increase in the limit of detectivity, over the observations of Adams and Dunham, seemed to us feasible on account of the more efficient gratings and faster photographic plates now available. Realizing, however, that the 1962 opposition of Mars was quite unfavorable, it was decided to carry out only an exploratory attempt, with the view of studying the ultimate possibilities of the spectrograph and detectors, for a thorough study during the 1965 opposition. Accordingly, only four nights of observing time, when the opposition had passed, were given to this project. As has been announced previously (Spinrad, Münch, and Kaplan 1963), on one of these nights, April 12/13, 1963, we obtained a spectrogram of excellent quality which shows faint but unmistakable lines which have been ascribed to $\mathrm{H}_{2} \mathrm{O}$ in Mars' atmosphere. Clearly, it would have been highly desirable to have obtained a plate of the same quality before opposition, when the Martian $\mathrm{H}_{2} \mathrm{O}$ lines would have appeared displaced from the telluric lines in the opposite direction. Since the single plate we did obtain, however, not only shows the sought-after water-vapor absorption, but also the $5 \nu_{3}$ band of $\mathrm{CO}_{2}$ at $\lambda 8700 \AA$, which allows us to make a direct estimate of the total pressure at the base of the Martian atmosphere, we feel that it is a matter of importance to discuss the results now, rather than to wait for the next Mars opposition.

\section{THE OBSERVATIONS}

The highest dispersion accessible with the 100-inch coude spectograph at wavelengths longer than $\lambda 8500 \AA$ is obtained with a 114-inch camera, in the first order of a grating of 600 lines $/ \mathrm{mm}$. For wavelengths shorter than $\lambda 8400 \AA$, a second-order grating with 900 lines/mm may be used to obtain a dispersion three times higher. Since the collimator focal length is $12 \mathrm{ft}$, resolving powers exceeding $10^{5}$ can be reached if a projected slit width around $10 \mu$ is used. The granularity of the I- $N$ type emulsion, however, does not effectively utilize such large resolving powers, and the higher accutance of the IV- $N$ type is required, if its slower speed can be overcome by increased exposures or strong hypersensitization. Test exposures of Mars and the Moon were carried out with the 900 -line/mm grating and a 73-inch camera on hypersensitized I- $N$ plates, on April 11/12, 1963. The high relative humidity prevailing on this night produced plates unsuitable for our primary purpose, although they confirmed our belief that the higher contrast of the IV- $N$ plate was indispensable for the success of the experiment. Accordingly, on the following night, April 12/13, 1963, we exposed to Mars a strongly hypersensitized IV- $N$ emulsion with the 114-inch camera and the 600-line/mm grating, for 270 minutes, between 02:55 and 07:25 (April 13) U.T. The width of the entrance slit was set at $17 \mu$, corresponding to $0.073 \AA$ in projection, and through the image rotator the direction of the slit was fixed visually along the Martian poles. The seeing conditions were only average (1-2 in a scale of 5), but a small north cap and a slight south polar hood were distinguishable. The appearance of the planet on small-scale direct photographs was normal. A northern cool wind prevailing for 


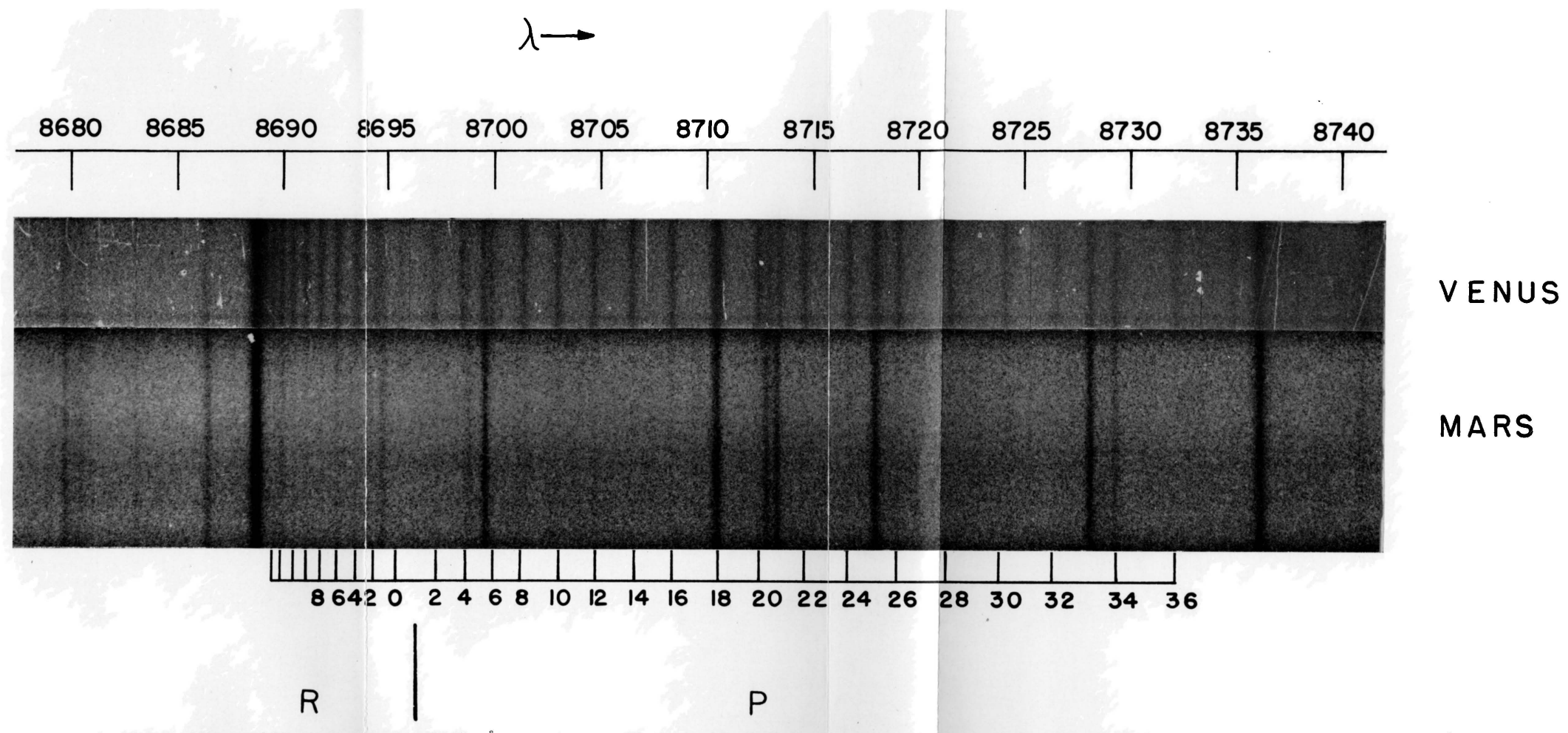

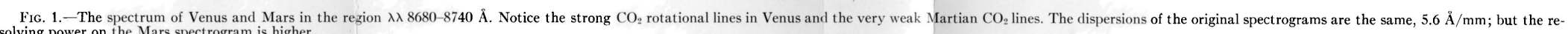
solving power on the Mars spectrogram is higher. 
some 12 hours before the exposure lowered the relative humidity considerably from its value during the preceding night. According to radio sonde data provided by the U.S. Weather Bureau, at $00^{\mathrm{h}}$ U.T. (April 13), the water content of the Earth's atmosphere, above Mount Wilson, was less than $5 \mathrm{~mm}$, a value well below average. The effective speed of the hypersensitized $I V-N$ plate was underestimated, for the density of the Mars plate we obtained is higher than optimum for spectrophotometry. Nonetheless, the definition of the plate is excellent and it shows clearly Fraunhofer lines listed by Babcock and Moore (1947): with intensities of -1 . As a further comparison it may be said that on this Mars spectrogram, almost every detail seen in the Utrecht Atlas of the Solar Spectrum (1940) can be detected. For example, a predicted Si I or Fe I line at $\lambda 8667.366 \AA$ is readily identified in the Mars spectrum. In the Utrecht Atlas it has a depth of only 0.04 and an equivalent width of $7 \mathrm{~mA}$. In the spectrogram of Venus, taken from the Mount Wilson plate collection, reproduced in Figure 1, this line cannot be seen. After comparing our plate with other ones found in the Mount Wilson files, we have convinced ourselves that ours is the spectrogram of Mars with the highest resolving power ever taken. It should be said, however, that almost immediately after the exposure of April 12 on Mars was completed, the sky became overcast and prevented the taking of a comparison spectrum on the Moon, as had been planned. A later attempt to obtain another spectrogram of Mars was carried out on June 9, 1963, again with the 100-inch telescope, but the humidity on this date was high and the plate came out somewhat underexposed for the $\lambda 8700 \AA$ region. The results to be presented in the following sections are, then, based exclusively on a single plate.

\section{MEASUREMENTS}

\section{a) The $\mathrm{CO}_{2}$ Lines}

The visual inspection of our Mars spectrogram in the $\lambda 8700 \AA$ region immediately revealed the presence of a number of faint lines, not present in the solar spectrum, near the position expected for rotational lines in the $R$ - and $P$-branches of the $5 \nu_{3}$ band of $\mathrm{CO}_{2}$. These lines are near the threshold of detectability for the plate, and were not a number of them close together giving to the eye the image of a whole pattern, it would be difficult to illustrate their presence convincingly, as has been done in Figure 1. The problem of measuring their wavelengths precisely was made difficult by their faintness and also by the presence of a number of faint Fraunhofer lines producing blends. In the $R$-branch, the lines $J=4$ and 6 and $J \geq 14$, are blended with solar lines at $\lambda \lambda 8693.958$ (S, Int 0), 8693.15 (S?, Int -3), 8692.342 ( $\mathrm{Ti}$, Int -2), and 8689.788 ( $\mathrm{Fe} p$, Int 0 ). Actually, lines of Rowland intensity -2 or -3 would not be detected if they were unblended, but when two such equally faint lines are blended, their combined intensity rises above the threshold set by plate grain. As far as simple detection is concerned, this blending effect is useful as a sort of local pre-sensitization. But, of course, a measure of the true intensity of such a line cannot be accomplished unless elaborate calibration techniques are employed. In our case, the lines $J=8,10$, and 12 , in the $R$-branch of the $5 \nu_{3}$ band are the strongest unblended ones, and their measured wavelengths agree satisfactorily with the laboratory wavelengths (Herzberg and Herzberg 1953). Some unblended lines of the $P$-branch can also be marginally detected, but appear weaker than the three lines of the $R$-branch referred to above. Conventional procedures to measure equivalent widths of absorption lines, when applied to features as weak as those we are concerned with, cannot be expected to provide results with the customary accuracy of photographic photometry. Nevertheless, because an estimate of their strength is of importance for the subsequent analysis, we have proceeded as follows. Microdensitometer tracings of the regions of interest were made, at a magnification of $\times 130$, for three non-overlapping strips of the spectrum separately. Since there is a gradient of density in the spectrogram across the direction of dispersion, the tracing corresponding to the southern one-third of the 
spectrum, having the lower density, was chosen to carry out the measurement, the other two being used only to estimate the mean depth and width of the lines in reference to the grain-plate noise.

The actual tracing used for the equivalent width measurements is reproduced in Figure 2, where the influence of plate grain on the absorption of the unblended $\mathrm{CO}_{2}$ lines can be appreciated. From the areas of the smoothed-out contours, drawn with consideration of the effective instrumental profile, we obtained the equivalent widths $3.3,3.7$, and $4.4 \mathrm{~m} \AA$ for the $J=8,10$, and 12 lines, respectively. The spread in these values by no means is a measure of the uncertainty involved, for the measures of the three lines in a given tracing hardly are independent of each other. Earlier estimates made from tracings obtained under different conditions by one of the writers (H. S.), essentially agree with these results. For calculation of the $\mathrm{CO}_{2}$ amount in Section IV, we will use as equivalent width $0.005 \mathrm{~cm}^{-1}$, which corresponds to the average value of $3.8 \mathrm{~m} \AA$.

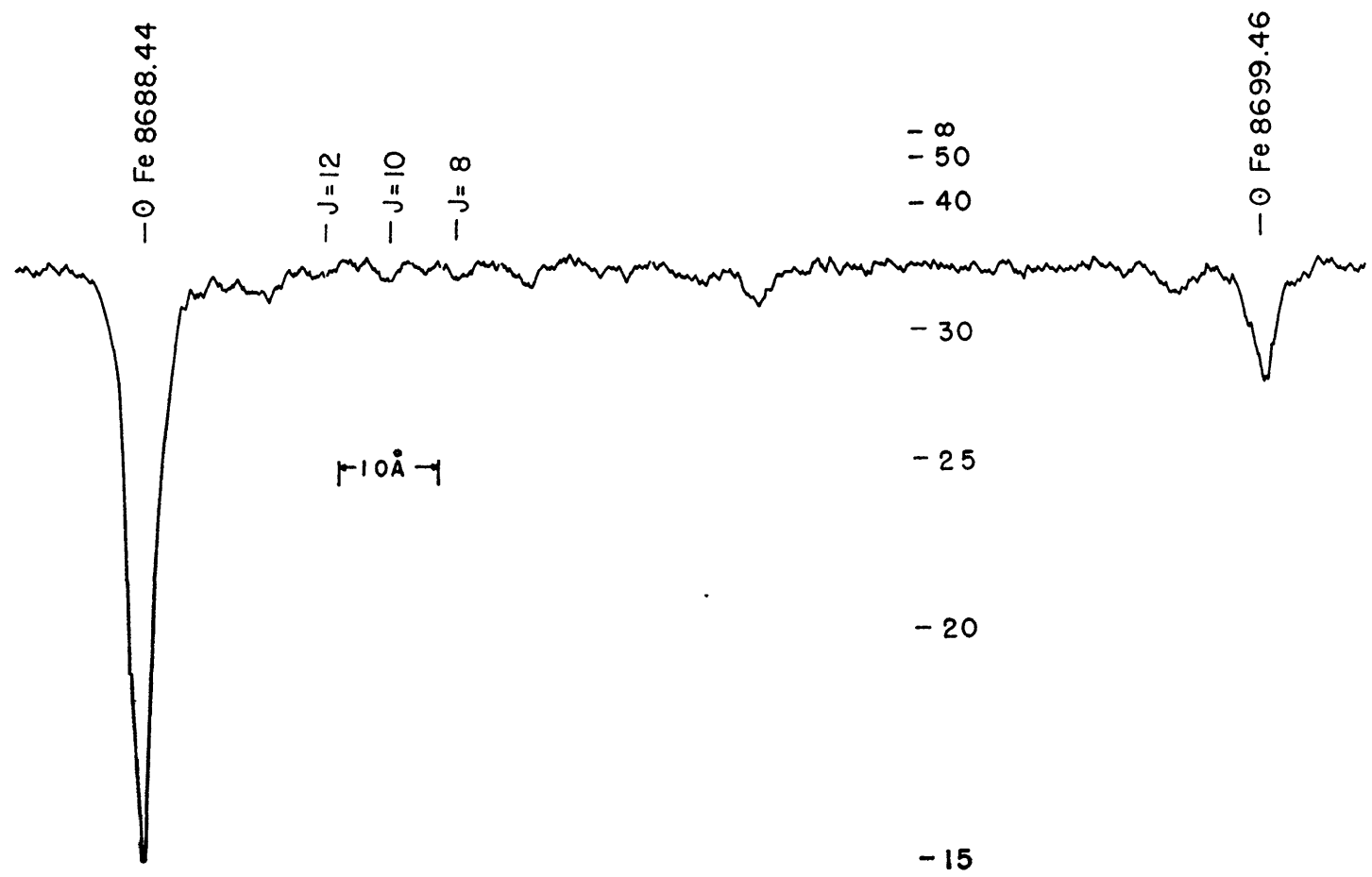

FIG. 2.-A microphotometer tracing of the $R$-branch in the $5 \nu_{3} \mathrm{CO}_{2}$ band of Mars. The measured lines $J=8,10,12$ and two solar lines are also shown. The non-linear scale of relative intensity is indicated.

In order to verify our sensitometric scale, drawn both from a wedge spectrogram and the step slit generally used at the Mount Wilson coudé, we have measured in our tracings the equivalent widths of a few medium-strong Fraunhofer lines and compared the results with the values derived from the Utrecht Atlas (1940). An estimate of the accuracy of our intensity measures of the weak $\mathrm{CO}_{2}$ absorption has been set by noticing that in our plate the $J=8,10$, or 12 lines appear slightly weaker than the Fraunhofer line at $\lambda 8667.376$ referred to above, which has an equivalent width of $7 \mathrm{~m} \AA$. We thus believe that the $\mathrm{CO}_{2}$ equivalent widths estimated before have an upper limit between 5 and $6 \mathrm{~m} \AA$.

\section{b) The $\mathrm{H}_{2} \mathrm{O}$ Lines}

The visual inspection of the Mars spectrum in the region of the $(2,1,1)$ band of $\mathrm{H}_{2} \mathrm{O}$, made independently by two of us (G. M. and H. S.), suggested the presence of 
faint longward components in a number of the stronger (telluric) lines in that band. The measurement of their displacements, with respect to the terrestrial lines, lead consistently to the value expected for lines originating in Mars's atmosphere, and on this basis the preliminary announcement for the detection of water vapor in Mars was made (Spinrad, Münch, and Kaplan 1963).

The lines we have ascribed to water vapor in Mars seem to be just about as weak as those of $\mathrm{CO}_{2}$ discussed above. However, it is not possible to illustrate their appearance as easily as it has been done in Figures 1 or 2 , because the $\lambda 8300 \AA$ band of $\mathrm{H}_{2} \mathrm{O}$ has a highly complicated structure, with its strong members well separated from each other and spread out over an interval more than $150 \AA$ wide. We believe that if the lines of the $5 \nu_{3}$ band of $\mathrm{CO}_{2}$ were 10 or $20 \AA$ from each other, in a spectral region densely crowded with lines, not only would it be difficult to illustrate convincingly their presence, but they might not have been discovered at all. Once the presence of the Martian water-vapor lines has been visually ascertained, their presence in microphotometer tracings becomes apparent, although the tracings themselves may not be a convincing proof for the existence of the lines.

TABLE 1

TELLURIC $\mathrm{H}_{2} \mathrm{O}$ Lines MEASURED FOR MARTIAN COMPONENTS

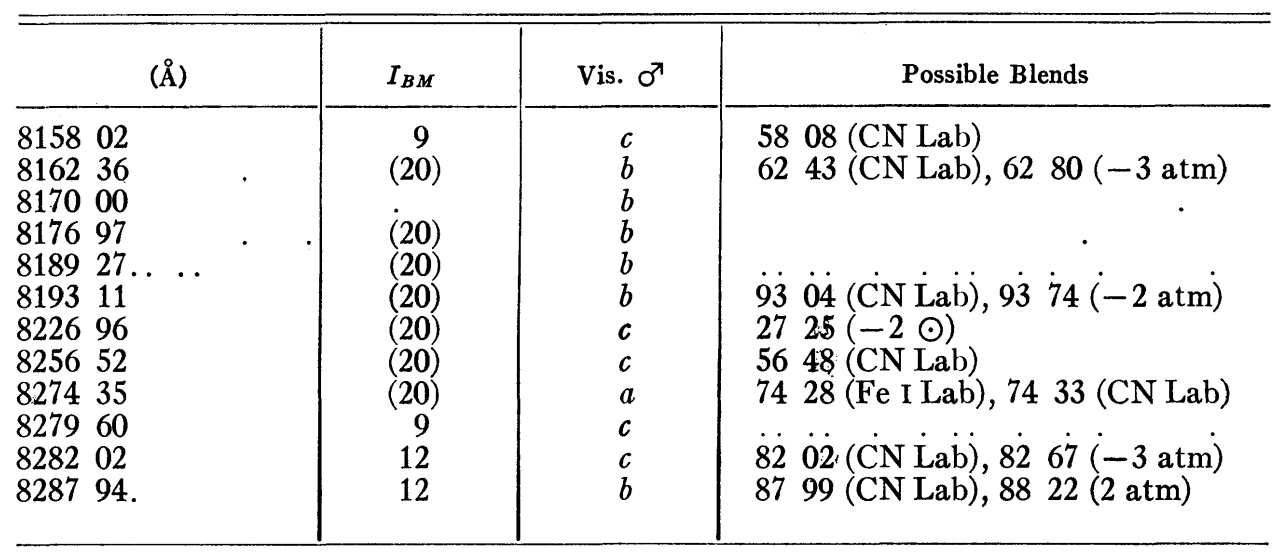

In our preliminary announcement eleven lines were given as having detectable components shifted by the expected amount, but the question of possible blends with Fraunhofer or telluric lines was only briefly mentioned. We shall now consider the matter further, by referring in Table 1 to the same eleven lines listed according to their laboratory wavelength $\lambda_{0}$. The intensities of the telluric components $I_{B M}$ as estimated by Babcock and Moore (1947) are given in the second column. The degree of visibility of the Martian component is indicated next by the letters $a, b$, and $c$, with " $a$ " qualifying those more easily detected or best defined in our plate, while " $c$ " stands for those just in the limit of visibility. In the last column are given the wavelengths and intensities of possible blends near the expected position of the Martian lines (displaced $0.41 \AA$ to the red of the laboratory system). In judging whether a line is actually affected by a blend or not, it should be kept in mind that in the solar spectrum, as obtained through the Earth's atmosphere, weak Fraunhofer lines lying within strong telluric lines cannot ever be seen. The Doppler shift of Mars, or of any other planet, can, however, shift such a line to a region free of telluric absorption. Recalling that nearly one-half of the weak Fraunhofer lines are unidentified, clearly we cannot be completely certain, but only in a probabilistic sense, that the weak components we observe in the Mars spectrum actually originate in Mars's atmosphere. Among the $\mathrm{H}_{2} \mathrm{O}$ lines listed in Table 1 the only one which is undoubtedly affected by a Fraunhofer line normally hidden behind the telluric $\mathrm{H}_{2} \mathrm{O}$ absorption, is that at $\lambda_{0}=8274.35 \AA$, 
since the laboratory spectrum of Fe I has a line at $8274.28 \AA$, in multiplet No. 1332 , and the other line of this multiplet at $8264.27 \AA$ can be seen in our plate. Also in spectra of Venus this line has been observed by one of us (H. S.) displaced both to the red and to the violet, and has been seen also in the spectrum of Jupiter. This line, then, does not provide evidence for the existence of a water-vapor line arising in Mars. Near the expected position for Martian components of the lines at 8158.02 and 8256.52 , are found solar lines of intensity -3. But in our spectrum they would appear Dopplershifted by an unadmissibly large amount, not to mention that it is very doubtful whether lines with such low intensity can be seen in our plate. These two lines, then, are not affected by blends. In our preliminary announcement we left open the question of possible blends with Fraunhofer lines due to the $(2,0)$ band of $\mathrm{CN}$, which in the laboratory shows numerous lines in this region. In a recent discussion (Rigutti and Drago-Chiuderi 1963) of the laboratory spectrum of CN, we noticed several lines whose wavelengths in the laboratory coincide with the telluric $\mathrm{H}_{2} \mathrm{O} \lambda_{0}$ 's. Whether the $\mathrm{CN}$ lines are strong enough to appear at our resolution is not known, but we have been conservative enough to include them as possible blends anyway.

It remains to consider the question of blends with weak telluric lines. In this regard the advantage of having obtained the plate under conditions of low humidity is fully realized. The strongly saturated lines listed in Table 1 appear in our plate sensibly narrower and weaker than in the Utrecht Atlas. The atmospheric lines suggested as possible blends, having intensities of -2 and -3 and being produced also by water vapor, are nearly unsaturated and would appear in our plate much weaker. For example, we mention the suggested blend to the line at $\lambda_{0}=8287.94$, to which an intensity of 2 is given by Babcock and Moore (1947). In the Utrecht Atlas this line shows quite strong on the wing of the line of intensity 12, but in our plate appears much weaker, with an intensity at most equal to a Fraunhofer line of intensity -1 . On these grounds, then, we are justified in considering the blends with atmospheric lines given by Babcock and Moore (1947) with intensities -2 and -3 as entirely negligible. Summarizing our discussion of the possible blends that might affect the components of the $\mathrm{H}_{2} \mathrm{O}$ lines considered to arise in the Martian atmosphere, we can say that, among those listed in Table 1 , only the lines at $\lambda$ 8274.35. and $\lambda 8287.94$ are really questionable on this account.

The procedure followed to estimate the equivalent widths of the unblended Martian components to the lines given in Table 1 is essentially the same as that described for the $\mathrm{CO}_{2}$ lines, and leads to results just as uncertain. In the case of the $\mathrm{H}_{2} \mathrm{O}$ lines we face the additional difficulties that the background set by the strong lines is varying rapidly, as is illustrated by the tracings reproduced in our preliminary announcement (Spinrad et al. 1963), and that our plate has a higher density in the $\lambda 8200$ region than at $\lambda 8700 \AA$, and effectively less contrast. In our plate the Martian $\mathrm{H}_{2} \mathrm{O}$ lines appear better defined in the part of the spectrum corresponding to the polar regions of the planet. This effect, not apparent in the $\mathrm{CO}_{2}$ lines, no doubt is in part a result of the curvature of the shoulder of the characteristic curve of the plate, but we are not certain that it is entirely an effect of contrast. Our material, however, does not warrant a quantitative discussion of this effect, and we simply have estimated the equivalent widths of the lines in tracings obtained with slits covering one-third of the planet's disk near the poles. The equivalent widths of all the Martian $\mathrm{H}_{2} \mathrm{O}$ lines were slightly greater at the south pole than at the north, but we do not know if the difference is significant. The variation of the values derived for the various lines is smaller than the uncertainties involved for which reason we can give only the value $4 \mathrm{~m} \AA$ or 0.006 $\mathrm{cm}^{-1}$ average equivalent width of the unblended lines at $\lambda 8176.97 \AA, \lambda 8189.27 \AA$, and $\lambda 8226.96 \AA$, which will be used in Section IV $b$ to determine the $\mathrm{H}_{2} \mathrm{O}$ abundance. 


\section{ABUNDANCE CALCULATIONS}

\section{a) $\mathrm{CO}_{2}$}

In Section III $a$ we derived an average equivalent width $W=0.005 \mathrm{~cm}^{-1}$ for the unblended lines $R 8, R 10$, and $R 12$ of the $5 \nu_{3}$ band of $\mathrm{CO}_{2}$. For such weak lines saturation is small and almost independent of pressure. It is therefore possible to use the measures to obtain a relatively unambiguous estimate of the $\mathrm{CO}_{2}$ abundance. The temperature dependence of the lines intensities, through the Boltzmann factor, is somewhat more important, and it would be desirable to have some idea of the atmospheric temperature. This could be obtained from accurate measurements of the relative equivalent widths of the individual rotational lines, which should follow a Boltzmann distribution. Unfortunately, the uncertainty of our measures and the blendings with Fraunhofer lines would make such a determination unreliable, but the apparent positions of the band maxima are consistent with an atmospheric temperature of about $230^{\circ} \mathrm{K}$.

From laboratory measurements kindly made for us by David Rank (1963), we estimate a band intensity of $0.63 \mathrm{~cm}^{-1} \mathrm{~km}$ atm at $300^{\circ} \mathrm{K}$, which would correspond to an average intensity $S=0.027 \mathrm{~cm}^{-1} \mathrm{~km}$ atm for the $R 8, R 10$, and $R 12$ lines at $230^{\circ} \mathrm{K}$. At $200^{\circ} \mathrm{K}$, the value would be $S=0.030 \mathrm{~cm}^{-1} \mathrm{~km}$ atm.

For absorption in the linear portion of the curve of growth, the amount of $\mathrm{CO}_{2}$ in the double traversal of the Martian atmosphere is simply given by the ratio between the equivalent width $W$ and the line strength $S$. If $w$ is the amount of $\mathrm{CO}_{2}$ along the vertical and $\eta$ is the effective air mass, we have for $T=230^{\circ} \mathrm{K}$

$$
\eta w=\frac{W}{S}=0.18 \mathrm{~km} \mathrm{~atm},
$$

where $\eta$ should be evaluated from the contribution of the air masses corresponding to the various points on the planetary disk represented in the microphotometer tracing. If $\theta \odot$ and $\theta_{\oplus}$ are the angles between the local normal of Mars and the directions of illumination and observation, we write

$$
\eta=\left\langle\sec \theta \odot+\sec \theta_{\oplus}\right\rangle,
$$

where the average indicated by the angular brackets should allow for the geometry of the situation, the smearing effects of guiding errors and seeing, and the non-linearity of the transmission of the spectrogram along the various regions covered by the analyzing slit of the microphotometer. Neglecting the small difference $\left(2^{\circ}\right)$ between the planetocentric latitudes of the subsolar and subearth points at the date of observation, for the meridional section covering the southern one-third of the planet, and for a smearing by guiding and seeing of 1 arcsec, we find $\eta=3.6$.

Actually, because of the narrowness of the lines there is some saturation, which may be accounted for by the expression for the equivalent width of a purely Dopplerbroadened line

$$
W=S \eta w\left\{1+\sum_{n=2}^{\infty} \frac{1}{n ! \sqrt{ } n}\left[-\frac{S \eta w}{\gamma_{D} \sqrt{ } \pi}\right]^{n-1}\right\}
$$

where $\gamma_{D}$ is the Doppler half-width. For a $\mathrm{CO}_{2}$ line at $\lambda 8700 \AA$ and $T=230^{\circ} \mathrm{K}$, $\gamma_{D}=0.011 \mathrm{~cm}^{-1}$, and with a table of the term in parentheses in equation (3) we find $\eta w=0.21 \mathrm{~km}$ atm. By considering the effect of collisional broadening on the curve of growth of the $\mathrm{CO}_{2}$ lines, we find from the tables by Harris (1948) that, for pressures between 20 and $100 \mathrm{mb}$ and a collisional half-width of about $0.1 \mathrm{~cm}^{-1}$ at STP, the 
value of $\eta w$ is somewhat reduced to $\eta w=0.20 \mathrm{~km}$ atm. The degree of saturation is still small enough that we may use the value $\eta=3.6$ given above, to find

$$
w\left(\mathrm{CO}_{2}\right)=55 \pm 20 \mathrm{~m} \text { atm }
$$

for $T=230^{\circ} \mathrm{K}$. At $T=200^{\circ} \mathrm{K}$, the corresponding number would be $50 \mathrm{~m}$ atm. The probable error attached to $w\left(\mathrm{CO}_{2}\right)$ results mainly from the uncertainty of our equivalent-width measurements.

\section{b) $\mathrm{H}_{2} \mathrm{O}$}

In Section III $b$ we derived an average equivalent width $W=0.006 \mathrm{~cm}^{-1}$ for the unblended $\mathrm{H}_{2} \mathrm{O}$ lines at $\lambda 8176.97 \AA, \lambda 8189.27 \AA$, and $\lambda 8226.96 \AA$, due to the transitions $3_{-3}-4_{-4}, 2_{-1}-3_{-2}$ and $2_{1}-2_{2}$, respectively. Rank (1963) has also measured for us the strengths of these lines: at $300^{\circ} \mathrm{K}$ their average intensity is $S=1.0 \mathrm{~cm}^{-1}$ per cm of precipitable water, which corresponds to an average intensity $S=1.3 \mathrm{~cm}^{-1}$ per precipitable $\mathrm{cm}$ at $230^{\circ} \mathrm{K}$.

The amount of $\mathrm{H}_{2} \mathrm{O}$ in the double traversal of the Martian atmosphere was determined in the same way as for $\mathrm{CO}_{2}$ in Section $\mathrm{IV} b$, with the result that $\eta w\left(\mathrm{H}_{2} \mathrm{O}\right)=50 \mu$. Again taking $\eta=3.6$, we obtain

$$
w\left(\mathrm{H}_{2} \mathrm{O}\right)=14 \pm 7 \mu .
$$

Our estimated relative error in the $\mathrm{H}_{2} \mathrm{O}$ amount is somewhat larger than for $\mathrm{CO}_{2}$ because of the greater uncertainty affecting the measurement of equivalent widths and line intensity.

$$
\text { c) } \mathrm{O}_{2}
$$

We did not detect any Doppler-shifted Martian components to the telluric lines in the $A$-band of $\mathrm{O}_{2}$ at about $\lambda 7600 \AA$. Because the telluric $\mathrm{O}_{2}$ lines are quite strong and broad, the detection of any weak Martian components is very difficult with photographic techniques, and we can therefore set only an upper limit to the Martian $\mathrm{O}_{2}$ content. By comparison with the $\mathrm{O}^{16} \mathrm{O}^{17}$ isotopic lines in the telluric spectrum appearing in our plate, we estimate that Doppler-shifted Martian lines would be visible if they were stronger than $20 \mathrm{~m} \AA$, or $W_{\sigma^{7}}\left(\mathrm{O}_{2}\right)<0.035 \mathrm{~cm}^{-1}$, in a tracing referring to the south polar region $(\eta \approx 4)$. The strength of the particular lines we are referring to can be derived from their observed equivalent widths in the telluric spectrum, and the expression

$$
W=2(S \bar{\gamma} \eta w)^{1 / 2}
$$

for a strong, isolated, pressure-broadened line with an effective collision half-width $\bar{\gamma}$. For a line near the apparent maximum of the $P$-branch, $W_{\oplus}\left(\mathrm{O}_{2}\right) \approx 1100 \mathrm{~m} \AA=1.9$ $\mathrm{cm}^{-1}$. According to Goody (1963), at STP $\gamma=0.047 \mathrm{~cm}^{-1}$. At the pressure and temperature at the base of the atmosphere above Mount Wilson this would correspond to $\gamma_{0}=0.040 \mathrm{~cm}^{-1}$. For a well-mixed atmosphere hydrostatically supported, in which the absorption coefficient is independent of temperature, the collision half-width $\bar{\gamma}$ is, according to the Curtis approximation (Kaplan 1959), $\gamma_{0} / 2=0.020 \mathrm{~cm}^{-1}$. With $w_{\oplus}\left(\mathrm{O}_{2}\right) \approx 1680 \mathrm{~m}$ atm above Mount Wilson, and for the air mass $\eta_{\oplus}=1.2$, we obtain from equation (6) $S=27 \mathrm{~cm}^{-1}$ per $\mathrm{km}$ atm. From the upper limit to the Martian $\mathrm{O}_{2}$ lines given above we then find $\eta w \sigma^{\top}\left(\mathrm{O}_{2}\right)<1.3 \mathrm{~m}$ atm, if the lines were unsaturated. Correcting for saturation, as was done in Section IVa for the $\mathrm{CO}_{2}$ lines, this upper limit is about doubled. The effective air mass for this degree of saturation would be slightly less than 4 , a value which gives

$$
w_{\sigma^{\top}}\left(\mathrm{O}_{2}\right) \leq 70 \mathrm{~cm} \text { atm. }
$$

This amount represents a threefold reduction to the upper limit given by Dunham (1952). 


\section{SURFACE PRESSURE}

A direct determination of the pressure at the base of the Martian atmosphere is of great intrinsic value because it provides a measure of the atmospheric mass and the key to an understanding of the atmospheric structure and circulation. It is also the main parameter entering critically in the pressing problem of aerodynamic entry and survival. The generally accepted value of the surface pressure is about $85 \mathrm{mb}$ (de Vaucouleurs 1954) based on various optical methods of which the most straightforward ones involve the assumption of a purely Rayleigh scattering atmosphere. Goody (1957) has pointed out that the presence of a persistent haze layer "might mean that existing estimates of the pressure are over-estimates," but no one seems to have suspected that the overestimate could be as large as our results will be seen to imply.

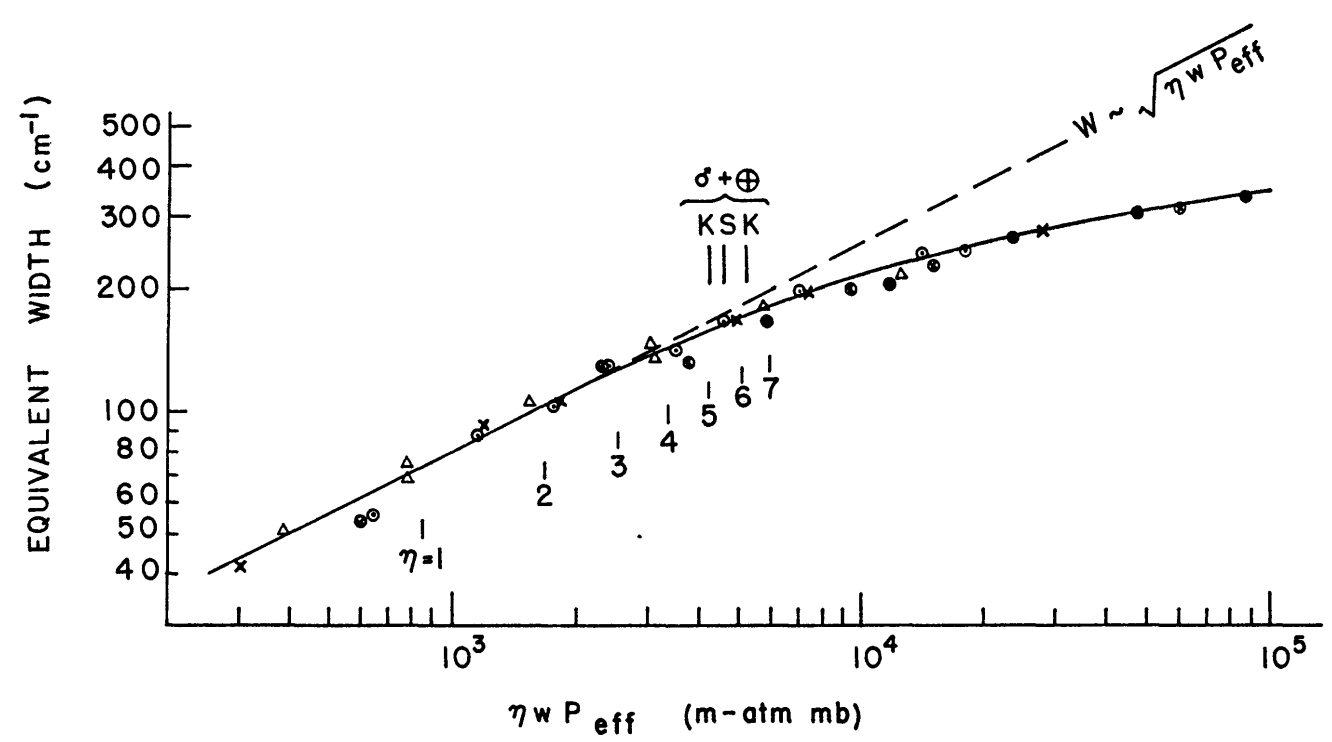

FIG. 3.-Equivalent width of $2-\mu$ band complex versus $\mathrm{CO}_{2}$ amount times effective pressure, on logarithmic scale, from data of Howard et al. (1955). Total pressure data as follows, in mm Hg: triangle, 35-50; circle with dot, 75-100; cross, 200; circle with cross, 400-470; solid circle, 760. Straight line represents "square-root law" absorption. Abscissa markings below curve for various telluric air-masses $\eta$, above curve for Earth plus Mars from Sinton $(S)$ and Kuiper upper and lower limits $(K)$.

The $\mathrm{CO}_{2}$ abundance $w_{\mathrm{CO}_{2}}=55 \pm 20 \mathrm{~m}$ atm, which we derived in Section IV $a$ from our measurements of the pressure-independent $5 \nu_{3}$ band, can now be used in conjunction with measurements of absorption by strongly pressure-dependent bands to determine the surface pressure. For this purpose we have used recent measurements by Sinton (1963) and Kuiper (1963) of the bands of $\mathrm{CO}_{2}$ around $2 \mu$, in particular the $4 \nu_{2}+\nu_{3}$ band at $2.06 \mu$ which is blended with telluric $\mathrm{H}_{2} \mathrm{O}$ to a smaller degree than are the high-frequency components.

The 2- $\mu$ bands are sufficiently strong that for both Mars and Earth the absorption is due to lines which are completely saturated at their centers. For bands of this type, the absorption is a function only of the product of the amount of absorber and effective pressure. This is illustrated in Figure 3, which shows the dependence of the equivalent width of the entire $2-\mu$ band complex on the product $w P_{\text {eff }}$, as derived from the data of Howard, Burch, and Williams (1955). The effective pressure $P_{\text {eff }}$, defined as the equivalent pressure at infinite dilution in $\mathrm{N}_{2}$, was obtained from the observation that self-broadening is 2.2 times as effective as nitrogen-broadening: that is,

$$
P_{\text {eff }}=P(1+1.2 a)
$$


where $a$ is the $\mathrm{CO}_{2}$ fraction by volume. As will be seen, the value of $w P_{\text {eff }}$ for Mars corresponds to a point on the straight-line portion of the curve, for which equation (6) is valid unless the pressure is so low that Doppler-broadening becomes important. Actually, the Martian lines are opaque out to several Doppler widths, so the band is effectively composed of strong collision-broadened lines and the above relations should hold. Grandjean and Goody (1955) have discussed this point for the weaker bands at $1.6 \mu$.

The measurements by Sinton were made at the 1960 opposition, when the Martian lines were not displaced from the telluric lines. Neglecting temperature dependences, the absorption by the combined Earth and Mars atmosphere is obtained by adding the contribution of the various $J$ lines, in the form

$$
A=\int\left(1-\exp \left\{-\left[\eta_{\oplus} \bar{P}_{\text {eff }}(\oplus) w_{\oplus}+\eta_{\sigma^{\top}} \bar{P}_{\text {eff }}\left(\sigma^{\top}\right) w_{\sigma^{\top}}\right] \sum_{J} \frac{\left(S_{J} \gamma_{s} / P_{s}\right)}{\pi\left(\nu-\nu_{J}\right)^{2}}\right\}\right) d \nu
$$

where $\gamma_{s}$ is the half-width at a standard pressure $P_{s}, \bar{P}_{\text {eff }}$ is, by the Curtis approximation (Kaplan 1959), half the effective surface pressure $P_{\text {eff }}$, and the integral is carried out over the whole band.

Sinton (1963) found the absorption in Mars at the center of the 2.06- $\mu$ band to be equivalent to an increase of 1.86 air masses in the solar spectrum over Mount Palomar. The effective surface pressure on Mars should therefore be given by

$$
P_{\text {eff }}\left(\sigma^{x}\right)=1.86 P_{\text {eff }}(\oplus) \frac{w_{\oplus}}{\eta_{\sigma^{\top}{ }^{w^{x}}}} .
$$

At Mount Palomar, $P_{\oplus} \approx 850 \mathrm{mb}$ and $w_{\oplus} \approx 2.2 \mathrm{~m}$ atm. Sinton implied a value of 2 for $\eta_{\sigma^{7}}$, which corresponds to measurements at the center of the disk. If we use the above values in equation (10) together with our value of $w_{\sigma^{7}}=55 \mathrm{~m}$ atm, we obtain $P_{\text {eff }}\left(\sigma^{7}\right)=32 \mathrm{mb}$. Equation (8) can then be used to correct for self-broadening in order to obtain the actual surface pressure, if $\mathrm{N}_{2}$ is assumed to be the broadening foreign gas.

The weight of a $55 \mathrm{~m}$ atm column of $\mathrm{CO}_{2}$ above a $\mathrm{cm}^{2}$ area on Mars is $4 \mathrm{mb}$; for a uniform mixture of $\mathrm{N}_{2}$ and $\mathrm{CO}_{2}$, this is equal to the fraction by weight times the surface pressure. Since the fraction by weight is equal to the fraction by volume, $a$, times the ratio of the molecular weight of $\mathrm{CO}_{2}$ to that of the mixture, we have

$$
4=\frac{44 a P_{\sigma^{7}}}{44 a+28(1-a)},
$$

which reduces formula (8) to

$$
P_{\text {eff }}\left(\sigma^{\nearrow}\right)=P_{\sigma^{T}}\left(1+\frac{33.6}{11 P_{\sigma^{\pi}}-16}\right),
$$

where all pressures have been expressed in $\mathrm{mb}$.

Argon is the only gas, besides $\mathrm{CO}_{2}$ and $\mathrm{N}_{2}$, likely to be present in appreciable amounts in the atmosphere of Mars. The presence of argon in the mixture would decrease $\gamma_{s}$ and increase $a$. The effects almost cancel one another, in such a way that formula (12) should also apply for a $\mathrm{N}_{2}-\mathrm{A}-\mathrm{CO}_{2}$ atmosphere.

The location of $w_{\sigma^{T}} \bar{P}_{\text {eff }}\left(\sigma^{\top}\right)$ is marked on Figure 3 . It is seen that the absorption falls well within the square-root region, indicating that the entire $2-\mu$-band complex is essentially composed of strong isolated lines.

If we introduce $P_{\text {eff }}\left(\sigma^{7}\right)=32 \mathrm{mb}$ into formula (12) we obtain for the surface pressure

$$
P_{\sigma^{7}}=29 \mathrm{mb} \text {. }
$$


The uncertainty of this estimate is of course larger than that of our estimate of $\mathrm{CO}_{2}$ abundance, because of the added uncertainties introduced by the use of Sinton's data. His measurements of Martian absorption show a large fluctuation, and the telluric absorption does not vary linearly with the square root of telluric air mass, as would be indicated by the data of Figure 3. The equivalent width of the $2.06-\mu$ band is less than that of the average of the other two components of the $2-\mu$ complex, and thus the square-root law should extend to even larger values of $\mu_{\oplus}$. Inaccuracies in the absorption measurements seem therefore to be present, probably due to the difficulty in determining the solar-spectrum envelope. However, since the matching of Martian versus telluric absorption was done by a direct comparison, the errors in absolute absorption should cancel to the first order and should have only a small effect on the accuracy of the derived pressure.

Fortunately, an independent estimate of the surface pressure can be made from measurements on the same band taken by Kuiper (1963) at McDonald Observatory on December 14, 1962. The spectral resolution was sufficient to begin to resolve the $P$ - and $R$-branches of the $2.06-\mu$ band, and the signal-to-noise ratio was high. Lunarspectrum envelopes were obtained; and comparison spectra were later made by passing sunlight, presumably at the same Earth air mass, $\eta_{\oplus}=1.05$, through laboratory $\mathrm{CO}_{2}$ in Tucson.

Kuiper kindly provided us with a copy of the Martian spectra and copies of comparison spectra with an 80-m geometric path of pure $\mathrm{CO}_{2}$ at pressures of 8.5 and 12 $\mathrm{cm} \mathrm{Hg}$. The apparent Martian absorption falls midway between those of the comparison spectra; by linear interpolation between our measurements of the apparent absorption we estimate a match at $80-\mathrm{m}$ geometric path length and $10.4 \mathrm{~cm} \mathrm{Hg}$. This corresponds to $w=11.1 \mathrm{~m}$ atm and $p=139 \mathrm{mb}$. For pure $\mathrm{CO}_{2}, P_{\text {eff }}=2.2 \times 139=306 \mathrm{mb}$, from formula (8).

Since these conditions are again such that absorption is proportional to $\left(\eta w P_{\text {eff }}\right)^{1 / 2}$, the effective Martian surface pressure can be obtained from

$$
P_{\text {eff }}\left(\sigma^{\top}\right)=2 P_{\text {eff }} \frac{w}{\eta_{\sigma^{7}} \sigma^{\pi}},
$$

where the factor of 2 is the result of again using the Curtis approximation to relate laboratory absorption to equivalent atmospheric absorption.

The measurements were made by putting the slit across the equatorial portion of Mars. If the absorption is proportional to $\eta^{n}$, the average air mass of an isotropically reflecting strip through the center of a uniformly illuminated hemisphere is

$$
\eta=2\left[\frac{\sqrt{ } \pi \Gamma(4-n / 2)}{(2-n) \Gamma(3-n / 2)}\right] \frac{1}{n} .
$$

For $n=\frac{1}{2}, \eta$ has the value 2.87, so at the phase angle of Mars on December 14, 1962, and for a slab of finite width, the average air mass should be about 3 or somewhat larger. If we use $\eta=3$ and $w_{\sigma^{7}}=55 \mathrm{~m}$ in equation (13) we obtain $P_{\text {eff }}\left(\sigma^{7}\right)=41 \mathrm{mb}$. This should be corrected for the temperature dependence of the line-width since the laboratory $\mathrm{CO}_{2}$ was presumably at room temperature. For a Martian temperature of $230^{\circ} \mathrm{K}, P_{\text {eff }}\left(\sigma^{7}\right)$ is reduced to about $37 \mathrm{mb}$, from which formula (12) can be used to obtain, for the surface pressure, $P_{\sigma^{7}}=34 \mathrm{mb}$. This value must be reduced somewhat hecause the Martian lines were Doppler-shifted relative to the telluric lines while of course the laboratory lines were not. However, the lines are sufficiently strong that Doppler-shifted pairs are saturated over the interval of Doppler-shifting, with the result that the required reduction is only about $1 \mathrm{mb}$.

A more straightforward estimate can be obtained by comparing the lunar spectra 
with the Martian spectra, although the envelopes appear to indicate a difference in the spectral variation of reflectivity between the Moon and Mars. The Doppler shift of the Mars lines tends to separate them from the telluric lines, but a rough calculation again shows that only a small correction would be required if the Doppler shift were ignored.

The spectral resolution obtained by Kuiper is sufficient that the signal maximum between the telluric bands at $2.01 \mu$ and $2.06 \mu$ should represent the true background. We took this as the background for both the lunar and Martian spectra in order to measure the equivalent widths of the $2.06-\mu$ band. The results are $W_{\oplus+\sigma^{r}}=35.4 \mathrm{~cm}^{-1}$ and $W_{\oplus}=18.6 \mathrm{~cm}^{-1}$.

Since the 2.06- $\mu$ band has approximately one-fourth of the equivalent width of the $2-\mu$-band complex, Figure 3 shows that the equivalent widths can be represented by equation (6). The Martian effective surface pressure can therefore be obtained from

$$
P_{\text {eff }}\left(\sigma^{\top}\right)=\frac{\eta_{\oplus} w_{\oplus} P_{\oplus}\left(W^{2} \sigma^{\top}+\oplus-W^{2}\right)}{\eta_{\sigma^{\top} w_{\sigma^{T}} W^{2}}} \text {. }
$$

At McDonald Observatory, $P_{\oplus}=800 \mathrm{mb}$ and $w_{\oplus} \approx 2.1 \mathrm{~m}$ atm. If we introduce in equation (15) these quantities, together with $\eta_{\oplus}=1.05, \eta_{\sigma^{\gamma}}=3, w_{\sigma^{\top}}=55 \mathrm{~m}$ atm, and the preceding values for the equivalent widths, we obtain $P_{\text {eff }}\left(\sigma^{7}\right)=28 \mathrm{mb}$. Introducing this into formula (12) we obtain $P_{\sigma^{7}}=25 \mathrm{mb}$ for the surface pressure.

This value and the one obtained by comparison with the laboratory data average almost exactly to that obtained from Sinton's data. Applying a small correction to the estimate from Kuiper's data because of the Doppler shift and to the estimate from Sinton's data because of the temperature and air-mass effects, we obtain for our estimate of the surface pressure of Mars

$$
P_{\sigma^{\top}}=25 \pm 15 \mathrm{mb} \text {. }
$$

We have also estimated the surface pressure from the observation by Kuiper (1952) that absorption by the 1.6- $\mu$ bands were enhanced to an amount equivalent to that by ten Earth air masses. We have followed the procedure of Grandjean and Goody (1955) to obtain $\eta_{\sigma^{7}}{ }_{0} P_{\text {eff }}\left(\sigma^{7}\right)$, except that we took $\gamma_{s}=0.07 \mathrm{~cm}^{-1}$ rather than their value of $\gamma_{s}=0.14 \mathrm{~cm}^{-1}$, which is too large. With $\eta_{\sigma^{\top}} \approx 4$ at the phase angle during Kuiper's observation (see Danielson, Gaustad, Schwarzschild, Weaver, and Woolf 1963 for an error in the Grandjean and Goody determination of $\eta$ ) and $w_{\sigma^{\gamma}}=55 \mathrm{~m}$, we obtain a surface pressure $P_{\sigma^{7}}=35 \mathrm{mb}$. This result is very sensitive, in fact approximately proportional, to the number of Earth air masses required to obtain a match. Unfortunately, the comparison is very difficult to make, since the absorption is weak and the solarspectrum envelope would be lowered at 10 air masses by telluric absorption. At the spectral resolution used, $\mathrm{CH}_{4}$ absorption on the low-frequency side would be particularly serious; on the high-frequency side, $\mathrm{H}_{2} \mathrm{O}$ absorption could give trouble. The effect of the lowering of the apparent solar envelope would be an overestimate of the number of Earth air masses necessary to match the Mars spectra. That this overestimate may have occurred is suggested by the small amount of apparent $\mathrm{CO}_{2}$ absorption. The equivalent width of the combined 1.6- $\mu$ bands on the Mars spectra appears to be about $7.5 \mathrm{~cm}^{-1}$. This can be compared with an equivalent width for the same bands of $12 \mathrm{~cm}^{-1}$ obtained by Howard et al. (1955) for a laboratory path of $2 \frac{1}{2}$ times the amount of $\mathrm{CO}_{2}$ above McDonald Observatory and a pressure $\frac{2}{3}$ as great. Thus the surface-pressure estimate would have to be revised downward to the extent that it would fall well within the limits given in our estimate from the recent and better spectra of Kuiper and Sinton.

Much higher surface pressures are obtained from the Stratoscope II results (Daniel- 
son et al. 1963). The equivalent width of the 2- $\mu$ bands on Mars obtained from Stratoscope was about $250 \mathrm{~cm}^{-1}$, after making a small correction for Martian $\mathrm{H}_{2} \mathrm{O}$. This value, together with $w=55 \mathrm{~m}$ atm and $\eta=3.5$, can be used to obtain an effective pressure from Figure 3. The result is a mean effective pressure of $80 \mathrm{mb}$, or an effective surface pressure of $160 \mathrm{mb}$. After applying a correction for temperature and selfbroadening, a surface pressure $P_{\sigma^{\pi}}=145 \mathrm{mb}$ is obtained.

The values of the surface pressure obtained from their measurements of the 2.7- $\mu$ bands are even higher. The data of Howard et al. (1955) do not extend to the equivalent width of $315 \mathrm{~cm}^{-1}$ obtained from Stratoscope, but they can be extrapolated to give a lower limit to the equivalent width and therefore the surface pressure. The result, again after application of a temperature correction, is $P_{\sigma^{7}}=350 \mathrm{mb}$.

These results are obviously completely in disagreement with each other, as well as with our value of $25 \pm 15 \mathrm{mb}$, and imply that Danielson et al. were unsuccessful in defining the solar-spectrum background or calibrating their detector or both. The apparent agreement obtained by Danielson et al. between the $\mathrm{CO}_{2}$ amount calculated from the two bands is accidental and is probably due to the use of different forms of empirical formulas representing the data of Howard et al. (1955). The high absorption measurements, particularly for the $2.7-\mu$ bands, were obtained only at pressures of $1 \mathrm{~atm}$, and the application of formulae of this type to different pressure conditions is dangerous, especially in the case of the $2.7-\mu$ bands for which the exponent of the $\mathrm{CO}_{2}$ amount is $2 \frac{1}{2}$ times as great as that of the pressure, in contrast to the equality of the exponents predicted by theory.

It should be mentioned that, because of the triangular appearance of the 2 - $\mu$-band complex in the Stratoscope spectra due to the low resolution, a reduction of the background by only 5 per cent would bring the derived surface pressure in agreement with our results. Such a reduction in the background is suggested, in fact, by their background continuum on the low-frequency side of the bands. However, the reduction of the background required to bring the $2.7-\mu$ band in agreement with our surface-pressure results is larger than seems reasonable.

The equivalent width of the Martian 2- $\mu$ bands as obtained from Stratoscope is considerably greater than our estimate of the combined telluric and Martian absorption from the spectra of Kuiper and Sinton. Kuiper's spectra in particular is much less ambiguous, especially for the 2.06- $\mu$ band. We feel, therefore, that we must ignore the Stratoscope results in our analysis.

Our estimate of the surface pressure of Mars, therefore, remains

$$
P_{\mathrm{o}^{\top}}=25 \pm 15 \mathrm{mb} \text {. }
$$

The uncertainties are due to those in our measurements of the $5 \nu_{3}$ band spectra and in Kuiper's and Sinton's measurements of the 2.06- $\mu$-band spectra.

\section{CONCLUDING REMARKS}

The $\mathrm{CO}_{2}$ content of the Martian atmosphere we have derived in this paper is, per se, not surprising, being only about twice as large as that suggested by Grandjean and Goody. What would appear indeed surprising is that the simple observation of the $5 \nu_{3}$ band of $\mathrm{CO}_{2}$ implies a value for the atmospheric pressure so low that $\mathrm{CO}_{2}$ itself becomes a major constituent. We emphasize that our determination, although rather uncertain on purely observational grounds, is straightforward and has as its basis simple physical principles, in contrast with previous estimates which were based on diverse assumptions practically impossible to verify observationally. The low value now suggested for the pressure prevailing in the atmosphere of Mars has important consequences, on which we shall comment briefly.

Let us first attempt to specify the abundances of the gases likely to be present in 
appreciable amounts in the Martian atmosphere. In Table 2 we consider the estimated mean values for the total surface pressure and partial pressure of $\mathrm{CO}_{2}$, together with the extreme values implied by the estimated range of uncertainty. Recalling that the larger $\mathrm{CO}_{2}$ amounts correspond to lower pressures, we see that for the low- and meanpressure models $\mathrm{CO}_{2}$ would indeed be a major constituent. The difference between the total pressure and the partial pressure of $\mathrm{CO}_{2}$ probably is made up by the contribution of the unobservable gases $\mathrm{A}$ and $\mathrm{N}_{2}$. Regarding argon we can make two assumptions: (a) The net $\mathrm{A}^{40}$ content of the Martian atmosphere is the same as that of the Earth (Brown 1952; Urey 1959). For a terrestrial abundance of 0.00934 per volume, the partial pressure in Mars would be $P_{a} \sigma^{7}(\mathrm{~A})=19 \mathrm{mb}$. (b) Alternatively we may assume that the net $\mathrm{A}^{40}$ content of Mars's atmosphere is to that of the Earth as the ratio of the corresponding planetary masses. Then we would have $P_{b} \mathrm{o}^{7}(\mathrm{~A})=2 \mathrm{mb}$.

From Table 2 we see that there is room for $19 \mathrm{mb}$ of radiogenic argon only in the medium- and high-pressure models. In the high-pressure model some $17 \mathrm{mb}$ could be allowed to $\mathrm{N}_{2}$, but in the mean model there would be hardly any $\mathrm{N}_{2}$. In the low-pressure

TABLE 2

Surface Pressures for the POSSIBle Major CONSTituents OF the MARTian AtMOSPHERE

\begin{tabular}{|c|c|c|c|}
\hline Pressure & $\begin{array}{l}\text { Low } \\
(\mathrm{mb})\end{array}$ & $\begin{array}{c}\text { Mean } \\
(\mathrm{mb})\end{array}$ & $\begin{array}{l}\text { High } \\
(\mathrm{mb})\end{array}$ \\
\hline $\begin{array}{ll}\text { Total } & \\
\mathrm{CO}_{2} \\
\mathrm{~A}^{40}: & a \\
& b \\
\mathrm{~N}_{2}: & a \\
& b\end{array}$ & $\begin{array}{r}10 \\
6 \\
2 \\
2 \\
2\end{array}$ & $\begin{array}{r}25 \\
4 \\
19 \\
2 \\
2 \\
19\end{array}$ & $\begin{array}{r}40 \\
3 \\
19 \\
2 \\
18 \\
38\end{array}$ \\
\hline
\end{tabular}

model obviously there could be no more than $4 \mathrm{mb}$ of $\mathrm{A}$ or $\mathrm{N}_{2}$. On the other hand, under the more logical hypothesis that the concentration of $\mathrm{K}^{40}$ in Mars is the same as in the Earth, and that degassing has occurred in both planets to the same extent, it would follow that the partial pressure of $\mathrm{N}_{2}$ is 2,19 , or $38 \mathrm{mb}$ for the three models. This possibility appears to us more attractive in the light of the ideas currently held for the origin of the atmospheres of the terrestrial planets. But still $\mathrm{N}_{2}$ would appear grossly underabundant with respect to the Earth's atmosphere, because, making the same assumption about the $\mathrm{N}_{2}$ content as was made in case $(b)$ above for $\mathrm{A}^{40}$, one obtains for Mars a nitrogen content of $300 \mathrm{gm} \mathrm{cm}^{-2}$ or a partial pressure of $120 \mathrm{mb}$. We would infer from these considerations, then, that the conjecture advanced by Urey (1959), regarding the likelihood of nitrogen escaping the atmosphere of Mars, is supported by our conclusions. It would then necessarily follow that the temperature prevailing at the layer where the escape is effectively taking place is in excess of $2000^{\circ} \mathrm{K}$.

The observational results presented in this paper have been seen to be affected by uncertainties which enter critically into the determination of the all-important value for the atmospheric pressure on Mars. Unlike the uncertainties affecting other methods used in the past to estimate that parameter, however, our determination can be much improved without essential difficulties. In short what is needed are numerous spectral observations, both in the photographic and the instrumental infrared, with increased resolving power and techniques to measure absorptions accurately. We hope to carry out an extensive observing program at the forthcoming presentation of Mars, using at the Mount Wilson coudé spectrograph a new grating giving at least twice the resolving power now attainable. The possibility of using photoelectric detectors, in addition to 
photographic plates, is now being explored, especially for observing the weak absorptions of $\mathrm{O}_{2}$ and $\mathrm{H}_{2} \mathrm{O}$. The observation of the $\mathrm{CO}_{2}$ bands in the instrumental infrared with increased resolution is just as important, and would appear to us practicable, if attention were paid to a restricted spectral range and enough time were given to such an observation.

It is a pleasure to express our thanks to Professor D. Rank for providing us with unpublished laboratory data, to Professor W. S. Benedict for discussions on the $\mathrm{H}_{2} \mathrm{O}$ spectrum, and to Professor G. P. Kuiper for sending us his observations in advance of publication.

\section{REFERENCES}

Adams, W. S. 1943, Ap. J., 93, 16.

Babcock, H. D., and Moore, C. 1947, The Solar Spectrum, $\lambda$ 6600- $\lambda 13495$ (Carnegie Institution of Washington Publ. 579; Washington, D.C.: Carnegie Institution of Washington).

Brown, H. 1952, The Atmospheres of the Earth and Planets, ed. G. P. Kuiper (Chicago: University of Chicago Press), p. 258.

Danielson, R. E., Gaustad, J. E., Schwarzschild, M., Weaver, H. F., and Woolf, N. J. 1963, A.J., in press.

Dollfus, A. 1961, Planets and Satellites, Vol. 3 : The Solar System, ed. G. P. Kuiper and B. Middlehurst (Chicago: University of Chicago Press), p. 381.

Dunham, T. 1952, The Atmospheres of the Earth and Planets, ed. G. P. Kuiper (Chicago: University of Chicago Press), p. 288.

Goody, R. M. 1957, Weather, 12, 3. 1963, Atmospheric Radiation (Oxford: Oxford University Press).

Grandjean, R., and Goody, R. M. 1955, Ap. J., 121, 548.

Harris, D. L. III. 1948, $A p$. J., 109, 53.

Herzberg, G. H., and Herzberg, L. 1953, J. Optical Soc. America, 43, 1039.

Hess, S. 1948, Pub. A.S.P., 60, 289.

1951, Planetary Atmospheric Project, Lowell Obs., Rept. No. 9, p. 172.

Howard, J. H., Burch, D. L., and Williams, D. 1955, Geophys. Res. Paper No. 40, AFCRL.

Kaplan, L. D. 1959, The Atmosphere and the Sea in Motion, ed. B. Bolin (New York: Rockefeller Institute Press), p. 170.

Kiess, C. C., Corliss, C. H., Kiess, H. K., and Corliss, E. L. R. 1957, Ap. J., 126, 579.

Kuiper, G. P. 1952, The Atmospheres of the Earth and Planets, ed. G. P. Kuiper (Chicago: University of Chicago Press), p. 362. 1963, Mém. Soc. R. Sci. Liège, 5th ser., Vol. 7

Lebedinskii, A. I., and Salova, G. I. 1962, Astr. J. USSR, 39, 494.

Rank, D. 1963, private communication.

Rigutti, M., and Drago-Chiuderi, F. 1963, Ann. d'ap., 26, 253.

Sagan, C. 1961, A.J., 66, 52.

Sinton, W. 1963, J. Quant. Spectrosc. Radiat. Transfer, 3, 551.

Spinrad, H., and Richardson, E. H. 1963, Icarus, $2,49$.

Spinrad, H., Münch, G., and Kaplan, L. D. 1963, A p. J., 137, 1319.

Urey, H. C. 1959, Hdb. d. Phys., ed. S. Flügge (Berlin: Springer-Verlag), 52, 400.

Vaucouleurs, G. de. 1954, Physics of the Planet Mars (London: Faber \& Faber), pp. 124, 219. 


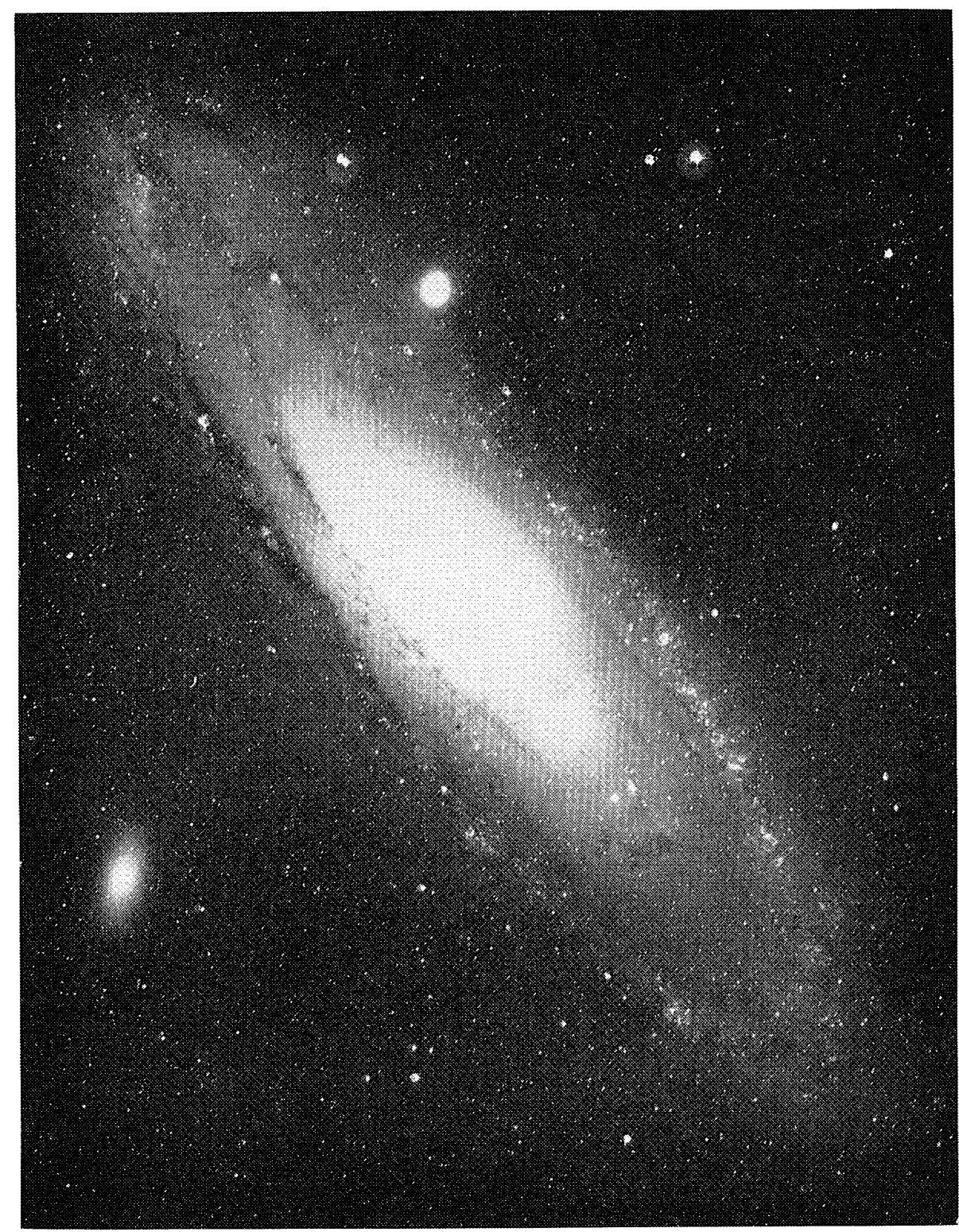

FIG. 9.-A photograph taken with the 48-inch Schmidt telescope through an $\mathrm{Ha}$ interference filter of $80 \AA$ half-width. The emission nebulae show best the apparent warping-up of the plane from the near side around to $\mathrm{M} 32$. 